\title{
Sparse preamble design for polarization division multiplexed CO-OFDM/OQAM channel estimation
}

\author{
Nhat-Quang Nhan, Pascal Morel, Stéphane Azou, Michel Morvan, Philippe Gravey, Erwan Pincemin
}

\begin{abstract}
In this paper, we consider the polarization division multiplexed (PDM) coherent optical - orthogonal frequency division multiplexing/offset-quadrature amplitude modulation (COOFDM/OQAM) system. The problem of channel estimation for this system is investigated. Firstly, we introduce the sparse preamble channel estimation method for PDM CO-OFDM/OQAM. We then propose a novel sparse preamble pattern. The interference approximation method is also taken into account for comparison. Simulation results, which are in accordance with the analysis, show that the proposed sparse preamble not only achieves significant gains in terms of bit-error rate (BER), but also offers lower peak-to-average power ratio (PAPR) and preamble overhead compared to the available solutions.
\end{abstract}

Index Terms-CO-OFDM/OQAM, coherent optical communications, polarization division multiplexing, channel estimation, preamble design.

\section{INTRODUCTION}

Multi-carrier modulations have recently attracted special attention from researchers to deal with the increasing capacity demands of optical fiber communication systems [1][4]. Above all, the offset-QAM for CO-OFDM modulation appears to be a promising solution to improve the spectral efficiency. The CO-OFDM/OQAM systems carefully localize the pulses in time and frequency-domains by using the pulse shaping filter bank and the staggered OQAM symbols (i.e., real symbols at twice the conventional OFDM/QAM symbol rate). This improved localization enhances system robustness against frequency offsets. Additionally, thanks to the good time localization, CO-OFDM/OQAM can avoid the use of cyclic prefix. This leads to gains in terms of system throughput of CO-OFDM/OQAM compared to its conventional counterpart. In addition, CO-OFDM/OQAM exhibits significantly low spectral side lobes, which allow to minimize guard bands between sub-carriers and hence to further improve spectral efficiency. For more details on offset-QAM multicarrier modulation, the readers can refer to [5], [6].

In CO-OFDM/OQAM systems, the sub-carrier functions are orthogonal in the real field only due to the presence

N.-Q. Nhan, P. Morel and S. Azou are with École nationale d'ingénieurs de Brest, Université Bretagne Loire, Lab-STICC, CNRS UMR 6285, France (e-mail: nhatquang.ee@gmail.com, pascal.morel@enib.fr, stephane.azou@enib.fr).

M. Morvan and P. Gravey are with IMT Atlantique Bretagne-Pays de la Loire, Université Bretagne Loire, Lab-STICC, CNRS UMR 6285, France (e-mail: michel.morvan@ telecom-bretagne.eu, philippe.gravey@telecombretagne.eu).

E. Pincemin is with the Orange Labs Networks, 22307 Lannion, France (e-mail: erwan.pincemin@orange.com).

Copyright (c) 2015 IEEE. Personal use of this material is permitted However, permission to use this material for any other purposes must be obtained from the IEEE by sending a request to pubs-permissions@ieee.org. of a specific filter bank applied on the sub-carriers. As a consequence, there exists intrinsic imaginary interference (IMI) among the neighboring sub-carriers (frequency-domain) and symbols (time-domain). Being interfered by the IMI, the estimation and compensation of channel dispersions for COOFDM/OQAM systems becomes more complicated compared to the conventional CO-OFDM ones, especially when polarization division multiplexing (PDM) is taken into account. Therefore, the quest for an optimal channel estimation and equalization for PDM CO-OFDM/OQAM systems is crucial.

Recent researches in channel dispersion compensation for CO-OFDM/OQAM mainly focus on the equalizer design while assuming that the channel transfer function is known [7], [8] or perfectly estimated [9]. In [10] and [11], the estimation of channel response by using preamble was investigated. Both methods focus on the preamble design to minimize the effect of IMI by allocating zeros around the target pilots and count on interpolation to find the channel response at the sub-carriers associated with the zero pilots. In contrast, there exists a channel estimation method, called Interference approximation method (IAM), that even exploits IMI as an advantage. Indeed, IAM is a well-known channel estimation method for OFDM/OQAM, which was originally proposed for radio communications in [12], [13]. This method was introduced for PDM CO-OFDM/OQAM in [14]-[16]. In fact, the shifted versions of the CO-OFDM/OQAM prototype filter in frequency and time domains can be viewed as a grid of filters for different frequency-time (FT) positions. To recover the symbol at a given FT position, the filter at that position must be slided and multiplied with all filters in the filter grid before summed up. Since the filters are designed to be orthogonal in real field only, the multiplication between filters in different FT locations result in a imaginary value called IMI coefficients. Fortunately, the pattern of IMI coefficients in the FT grid is unchanged for any reference FT position. The IAM method relies on this characteristic to design the preamble.

There are several variances of IAM with different preamble patterns. Unfortunately, only the IAM pattern with real pilots (IAM-R) was considered in [14]-[16]. In this paper, we show that another IAM pattern, namely the enhanced IAM with complex pilots (E-IAM-C), can also be applied for PDM COOFDM/OQAM systems with remarkable BER performance improvement. We then point out that, although the E-IAM$\mathrm{C}$ offers a significant gain in BER compared to the IAM-R, it however results in a high value of PAPR. This is the motivation for the following main contributions of this paper. Firstly, we introduce the sparse preamble (SP) channel estimation (CE) for PDM CO-OFDM/OQAM systems. The SP CE requires a 
lower preamble overhead than the IAM methods. Secondly, we propose a novel SP design for these systems. The proposed solution achieves a gain in BER with a lower PAPR compared to the E-IAM-C method. In comparison with the IAM-R method in [14], the proposed SP design attains a significant gain in BER with an equivalent PAPR.

The notations in this paper are as follows. Vectors are denoted by lower-case bold letters, while matrices are denoted by upper-case bold letters. $\|\cdot\|$ stands for the $L^{2}$ norm (of a vector) or Frobenius norm (of a matrix). (.)* is the complex conjugate. $(\cdot)^{T}$ represents the matrix transpose, while the conjugate transpose of a matrix is $(\cdot)^{\dagger}$. The Kronecker product is denoted by $\otimes .\langle\cdot, \cdot\rangle$ stands for the inner product operator. The identity matrix of size $k$ is denoted by $\mathbf{I}_{k}$.

\section{Preliminary}

\section{A. System model}

Let us consider a system model as shown in Fig. 1. Denote by $M, m$ and $T$ the number of sub-carriers, the sub-carrier index and the QAM symbol duration, respectively. At the transmitter side, on $x$-polarization, a complex-to-real $(\mathrm{C} 2 \mathrm{R})$ converter is used at each sub-carrier $m$ to convert the complexvalued QAM input symbols $c_{m}^{x}$ into real-valued symbols $a_{x, m, n}$, which has symbol duration $T / 2$ indexed by $n$. The symbols $a_{x, m, n}$ are then fed to the synthesis filter bank (SFB) generated by a prototype filter $G(t)$ with overlapping factor $K$. The CO-OFDM/OQAM signal on $x$-polarization is found by summing up the outputs from SFB. Similar process is applied to find CO-OFDM/OQAM signal on $y$-polarization. The dual-polarized signal is then passed through optical fiber. At the receiver side, after adding the additive noise $w$, the received signal is split into two polarizations. Analysis filter bank (AFB) is then applied on each polarization to reconstruct the OQAM signals. At this point, one should note that, even if SFB and AFB are directly connected (back-to-back without additive noise), the output signals from AFB are always complex since the reconstruction is perfect in real field only. The imaginary parts, which are caused by the nonorthogonality of the OFDM-OQAM prototype filter in imaginary field, come from the neighboring frequency-time symbols. The complex output signals from AFB pass the channel estimation and equalization block, where the channel response is estimated and equalized in frequency domain by using the single-tap equalizer. The real-valued OQAM symbols can be estimated by taking only real part of the equalized signals. The estimated OQAM symbols are then converted to the estimated QAM signals thanks to the real-to-complex (R2C) converters at QAM conversion block. In practice, the SFB and AFB are efficiently implemented by fast Fourier transform (FFT) with the polyphase network (PPN) approach [6]. In this paper, we present the continuous-time model for the purpose of system analysis.

Denote $g_{m, n}(t)=\theta_{m, n} g\left(t-n \frac{T}{2}\right) e^{j 2 \pi m \Delta_{f} t}$, where $\theta_{m, n}=$ $j^{m+n}$ and $\Delta_{f}$ stands for the frequency spacing between two successive sub-carriers. For each polarization, the baseband continuous-time CO-OFDM/OQAM signal at output of SFB reads

$$
s(t)=\sum_{n=1}^{N} \sum_{m=0}^{M-1} a_{m, n} g_{m, n}(t),
$$

where $N$ stands for number of CO-OFDM/OQAM blocks. $M$ is the number of sub-carriers. $a_{m, n}$ is the real-valued transmitted symbol at the frequency-time (FT) location $(m, n)$. $g_{m, n}(t)$ is the shifted version of prototype filter $G(t)$ in both frequency and time domains. Denote $z(t)$ the input of AFB (see Fig. 1). Assuming the ideal condition such that $z(t)=s(t)$, the symbol $t_{m, n}$ at output of the AFB is defined by

$$
t_{m, n}=\left\langle g_{m, n}, s\right\rangle=\int_{-\infty}^{\infty} g_{m, n}^{*}(t) s(t) d t .
$$

Since $g_{m, n}(t)$ is designed to be orthogonal in real field only, there always exists the imaginary interferences between filters at different FT locations, i.e., $\left\langle g_{m, n}, g_{m+p, n+q}\right\rangle=$ $j\langle g\rangle_{m, n}^{m+p, n+q}$, where $\langle g\rangle_{m, n}^{m+p, n+q}$ is a real-valued coefficient, which could be computed in advance for a given prototype filter. Hence, one can deduce from (1) and (2) that

$$
t_{m, n}=a_{m, n}+j a_{m, n}^{(i)},
$$

where $a_{x, m, n}^{(i)}=\sum_{(p, q) \in \Omega_{1,1}^{*}} a_{x, m+p, n+q}\langle g\rangle_{m, n}^{m+p, n+q}$. In which, $\Omega_{1,1}^{*}=\{(p, q) \neq(0,0)|| p|\leq 1| q \mid, \leq 1\}$. This implies the common assumption that IMI only comes from the first-order neighbors of the FT location $(m, n)$. For a given prototype filter, the first-order IMI coefficients $j\langle g\rangle_{m, n}^{m+p, n+q},(p, q) \in$ $\Omega_{1,1}^{*}$, of a targeted symbol $a_{m, n}$ are constants and having the following format

$$
\left[\begin{array}{ccc}
j(-1)^{m} \delta & -j \beta & j(-1)^{m} \delta \\
-j(-1)^{m} \gamma & a_{m, n} & j(-1)^{m} \gamma \\
j(-1)^{m} \delta & j \beta & j(-1)^{m} \delta
\end{array}\right],
$$

where $\delta<\gamma<\beta$, the vertical and horizontal axes stand for the frequency and time dimensions, respectively. For the well-known PHYDYAS prototype filter, these coefficients are $\gamma=0.553, \beta=0.25$ and $\delta=0.2172$

Let us denote by $H_{x x, m, n}, H_{x y, m, n}, H_{y x, m, n}$ and $H_{y y, m, n}$ channel responses at FT location $(m, n)$ for polarizations $x$ and $y$, respectively. After passing through the fiber channel, the demodulated signal at FT location $(m, n)$ reads

$$
\underbrace{\left[\begin{array}{l}
r_{x, m, n} \\
r_{y, m, n}
\end{array}\right]}_{\mathbf{r}_{m, n}}=\underbrace{\left[\begin{array}{ll}
H_{x x, m, n} & H_{x y, m, n} \\
H_{y x, m, n} & H_{y y, m, n}
\end{array}\right]}_{\mathbf{H}_{m, n}} \underbrace{\left[\begin{array}{c}
t_{x, m, n} \\
t_{y, m, n}
\end{array}\right]}_{\mathbf{t}_{m, n}}+\underbrace{\left[\begin{array}{l}
w_{x, m, n} \\
w_{y, m, n}
\end{array}\right]}_{\mathbf{w}_{m, n}},
$$

where $t_{x, m, n}$ and $t_{y, m, n}$ are $t_{m, n}$ on $\mathrm{x}$ - and y-polarization, respectively. If the transmitted symbol $a_{m, n}$ is a pilot, the corresponding $t_{m, n}$ is then referred to as a pseudo pilot.

\section{B. Interference approximation method}

Let us consider the case where pilots in the preamble are located over all the sub-carriers (see Fig. 2). Denote $n_{1}$ and $n_{2}$ the two time positions, where the targeted pseudo pilots are placed. From (4), by assuming that $\mathbf{H}_{m, n_{1}}=\mathbf{H}_{m, n_{2}}=$ 


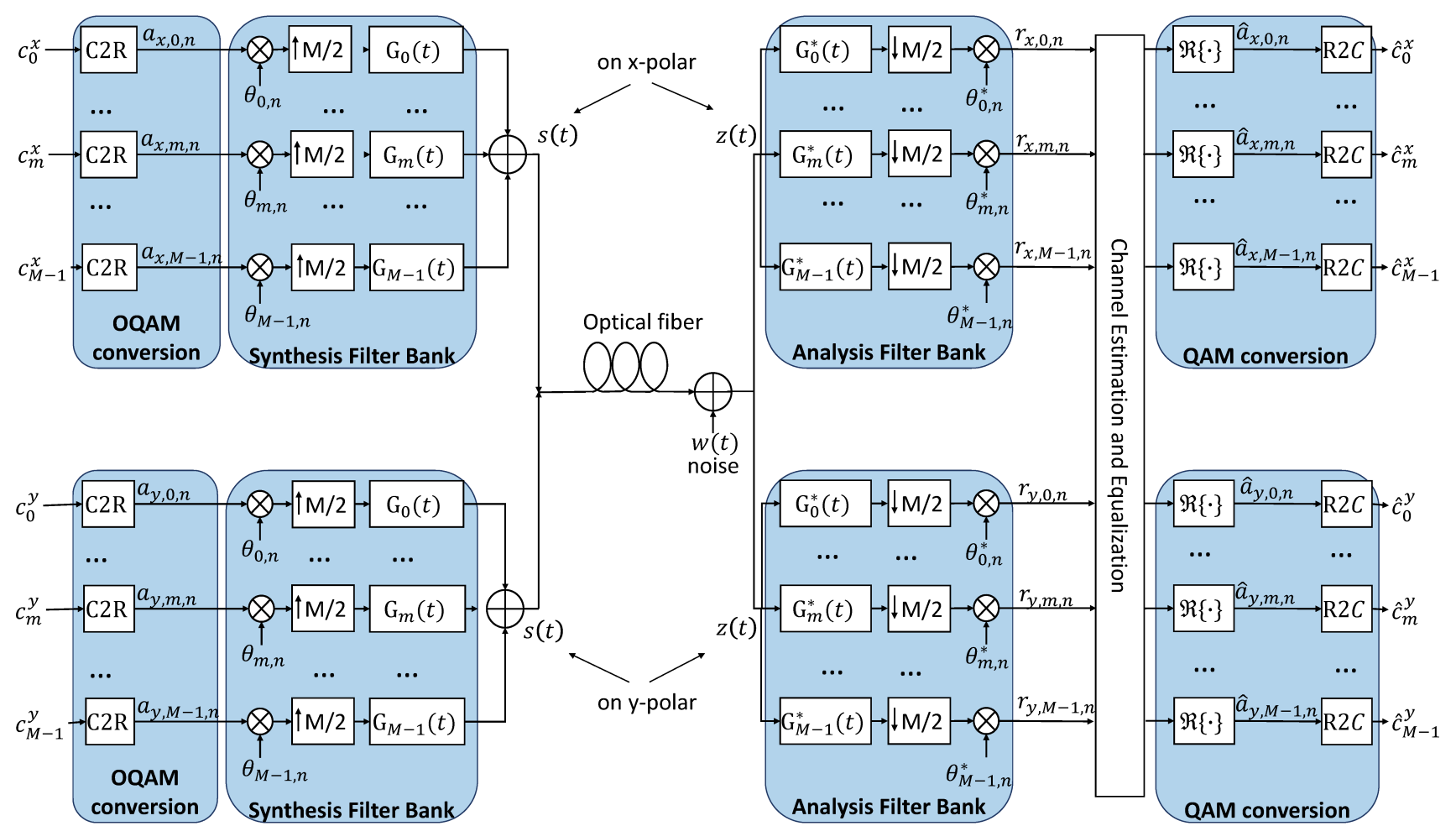

Fig. 1. System model.

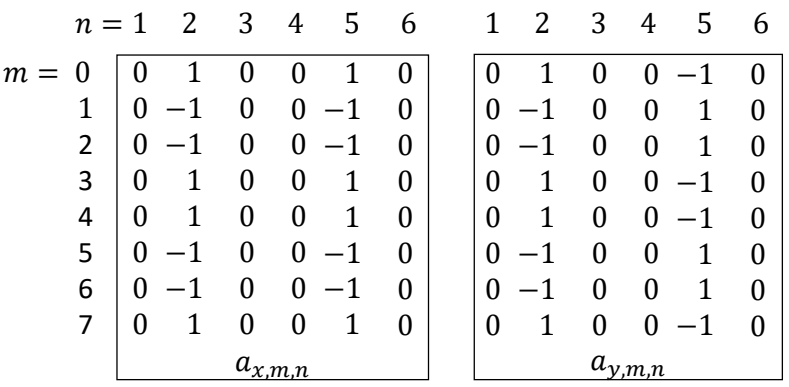

(a) IAM-R.

\begin{tabular}{|c|c|c|c|c|c|c|c|c|c|c|c|c|}
\hline$n$ & 1 & 2 & 3 & 4 & & & 1 & Z & 3 & 4 & 5 & $\epsilon$ \\
\hline$=0$ & $j$ & 1 & $-j$ & $j$ & 1 & $-j$ & $j$ & 1 & $-j$ & $-j$ & -1 & \\
\hline 1 & -1 & $-j$ & 1 & -1 & & 1 & $\mid-1$ & $-j$ & 1 & 1 & $j$ & -1 \\
\hline 2 & $-j$ & -1 & $j$ & $-j$ & & $j$ & $-j$ & -1 & $j$ & $j$ & 1 & $-j$ \\
\hline 3 & 1 & $j$ & -1 & 1 & $j$ & -1 & 1 & $j$ & -1 & -1 & $-j$ & 1 \\
\hline 4 & $j$ & 1 & $-j$ & $j$ & 1 & $-j$ & $j$ & 1 & $-j$ & $-j$ & -1 & $j$ \\
\hline 5 & -1 & $-j$ & 1 & -1 & & 1 & -1 & $-j$ & 1 & 1 & $j$ & -1 \\
\hline 6 & $-j$ & -1 & $j$ & $-j$ & -1 & $j$ & $-j$ & -1 & $j$ & $j$ & 1 & $-j$ \\
\hline 7 & $j$ & $j$ & -1 & & & -1 & $j$ & & -1 & & $-j$ & \\
\hline & $a_{x, r}$ & & & licate & & & & & & & & \\
\hline
\end{tabular}

(b) E-IAM-C.

Fig. 2. Examples of different preamble patterns for PDM CO-OFDM/OQAM with $M=8$ sub-carriers.

$\mathbf{H}_{m}$, the received signal can be represented in matrix form as follows

$$
\left[\begin{array}{l}
\mathbf{r}_{m, n_{1}} \\
\mathbf{r}_{m, n_{2}}
\end{array}\right]^{T}=\mathbf{H}_{m}\left[\begin{array}{ll}
t_{x, m, n_{1}} & t_{x, m, n_{2}} \\
t_{y, m, n_{1}} & t_{y, m, n_{2}}
\end{array}\right]+\left[\begin{array}{c}
\mathbf{w}_{m, n_{1}} \\
\mathbf{w}_{m, n_{2}}
\end{array}\right]^{T}
$$

The channel matrix $\mathbf{H}_{m}$ can then be estimated in frequency domain by using the least square method, which is optimal when $t_{x, m, n_{1}}=t_{x, m, n_{2}}=t_{y, m, n_{1}}=-t_{y, m, n_{2}}=t_{m}$. Finally, the estimated channel, denoted by $\hat{\mathbf{H}}_{m}$, reads

$$
\hat{\mathbf{H}}_{m}=\mathbf{H}_{m}+\left[\begin{array}{c}
\mathbf{w}_{m, n_{1}} \\
\mathbf{w}_{m, n_{2}}
\end{array}\right]^{T}\left[\begin{array}{cc}
1 & 1 \\
1 & -1
\end{array}\right] /\left(2 t_{m}\right) .
$$

One can deduce from (6) that mean square error (MSE) of the $\mathrm{CE}$ is given by $E\left(\left\|\mathbf{H}-\hat{\mathbf{H}}_{m}\right\|^{2}\right)=2 \sigma^{2} /\left|t_{m}\right|^{2}$, where $\sigma^{2}$ is the noise variance. This implies that the design of preamble pattern to optimize $t_{m}$ directly links to $\mathrm{CE}$ performance. Readily, the higher $\left|t_{m}\right|$, the smaller MSE for CE. Therefore, taking into account the pattern format in (3), the preamble pattern can be designed so as to maximize the magnitude of pseudo pilot $t_{m}$. This method is referred to as interference approximation method (IAM).

Several IAM preambles were proposed in [12], [13] namely IAM-R, IAM-C and E-IAM-C. Among those, the E-IAM-C gives a greater $\left|t_{m}\right|$ than the others. However, only IAM$\mathrm{R}$ was exploited in [14] for coherent optical communications. Therefore as an additional contribution of this paper, we show that the E-IAM-C could also be considered for coherent optical communications. Indeed, as shown in Fig. 2, the E-IAM-C pattern can be straightforwardly applied for PDM CO-OFDM/OQAM channel estimation by duplicating the triple of CO-OFDM/OQAM symbols in time domain for $x$ polarization and simultaneously duplicating and flipping signs of the triple of CO-OFDM/OQAM symbols in time domain for $y$-polarization. One should note that these preambles need 6 CO-OFDM/OQAM symbols (i.e., 6 time-slots) for each 
polarization. The pseudo pilots are counted at $n_{1}=2$ and $n_{2}=5$.

One should note that, in a pseudo pilot $t_{m, n}=a_{m, n}+$ $j a_{m, n}^{(i)}$, the actual pilot is $a_{m, n}$ and $j a_{m, n}^{(i)}$ is the IMI from the neighboring frequency-time symbols, which are caused by the nonorthogonality of the OFDM-OQAM prototype filter in imaginary field. The IAM-based channel estimation aims to maximize the amplitude of the pseudo pilot by designing the preamble pattern so as to exploit the IMI, while maintaining an equivalent power between the actual pilots and payload.

\section{ANALYSIS}

This section firstly introduces the application of sparse preamble channel estimation for PDM CO-OFDM/OQAM systems. Subsequently, a novel sparse-preamble based channel estimation method for these systems is proposed.

\section{A. Sparse preamble for PDM CO-OFDM/OQAM}

The SP CE method aims to estimate the truncated channel impulse responses (CIR) of length $L_{h}$ thanks to the sparsely distributed preamble pilots. On each polarization of the PDM CO-OFDM/OQAM systems, these pilots are grouped into two sets with $L_{h}$ pilots each. Pilots in the set $\mathcal{S}_{i}, i \in\{1,2\}$, are placed at the sub-carriers $\left\{p_{i}, p_{i}+N, p_{i}+2 N, \cdots, p_{i}+\left(L_{h}-1\right) N\right\}$, where $N$ is an integer and the starting position $p_{i} \in\{0,1, \cdots, N-1\}$. The rest of the sub-carriers are set to zeros. In other words, the pilots within each set are equispaced. To avoid interference, the non-zero pilots need to be separated by at least one zero. The minimum value of $N$ that satisfies this condition is $N=4$. Therefore, the required number of sub-carriers to estimate the CIR of length $L_{h}$ is $M=N L_{h}$, where $N \geq 4$.

Consider the SP CE at a targeted time position $n=n_{0}$ (e.g., $\left.n_{0}=2\right)$, the time index $n$ can then be dropped for simplicity. From (4), the received symbol vector at the sub-carrier $m$ can be rewritten as

$$
\mathbf{r}_{m}=\left[\begin{array}{ll}
H_{x x, m} & H_{x y, m} \\
H_{y x, m} & H_{y y, m}
\end{array}\right]\left[\begin{array}{c}
t_{x, m} \\
t_{y, m}
\end{array}\right]+\mathbf{w}_{m} .
$$

Denote $\mathbf{h}_{x x}, \mathbf{h}_{y y}, \mathbf{h}_{x y}, \mathbf{h}_{y x}$ the CD and PMD induced discrete time-domain CIR vectors of size $L_{h} \times 1$, and $\mathbf{F}$ the discrete Fourier transform (DFT) matrix of size $M$. Since the estimated $L_{h}$ is less than $M$, one can write

$$
\begin{aligned}
& H_{x x, m}=\mathbf{F}\left(m, 1: L_{h}\right) \mathbf{h}_{x x}, \quad H_{x y, m}=\mathbf{F}\left(m, 1: L_{h}\right) \mathbf{h}_{x y}, \\
& H_{y x, m}=\mathbf{F}\left(m, 1: L_{h}\right) \mathbf{h}_{y x}, \quad H_{y y, m}=\mathbf{F}\left(m, 1: L_{h}\right) \mathbf{h}_{y y}
\end{aligned}
$$

where $\mathbf{F}\left(m, 1: L_{h}\right)$, which is defined as in Matlab notation, stands for a vector taking the first $L_{h}$ elements from the $m^{\text {th }}$ row of $\mathbf{F}$. The received symbols from the set $i$ of preamble pilots read

$$
\mathbf{r}^{\left(p_{i}\right)}=\left[\mathbf{r}_{p_{i}}^{T}, \mathbf{r}_{p_{i}+N}^{T}, \cdots, \mathbf{r}_{p_{i}+\left(L_{h}-1\right) N}^{T}\right]^{T} .
$$

Define

$$
\begin{gathered}
\mathbf{G}_{m}=\mathbf{I}_{2} \otimes \mathbf{F}\left(m, 1: L_{h}\right) \\
\overline{\mathbf{F}}^{\left(p_{i}\right)}=\left[\mathbf{G}_{p_{i}}^{T}, \mathbf{G}_{p_{i}+N}^{T}, \cdots, \mathbf{G}_{p_{i}+\left(L_{h}-1\right) N}^{T}\right]^{T},
\end{gathered}
$$

$$
\begin{aligned}
& \mathbf{T}_{x}^{\left(p_{i}\right)}=\operatorname{diag}\left(\left[t_{x, p_{i}}, t_{x, p_{i}+N}, \cdots, t_{x, p_{i}+\left(L_{h}-1\right) N}\right]\right) \otimes \mathbf{I}_{2}, \\
& \mathbf{T}_{y}^{\left(p_{i}\right)}=\operatorname{diag}\left(\left[t_{y, p_{i}}, t_{y, p_{i}+N}, \cdots, t_{y, p_{i}+\left(L_{h}-1\right) N}\right]\right) \otimes \mathbf{I}_{2},
\end{aligned}
$$

and $\mathbf{h}^{(x)}=\left[\mathbf{h}_{x x}^{T}, \mathbf{h}_{y x}^{T}\right]^{T}, \mathbf{h}^{(y)}=\left[\mathbf{h}_{x y}^{T}, \mathbf{h}_{y y}^{T}\right]^{T}$. Substitution yields

$$
\mathbf{r}^{\left(p_{i}\right)}=\mathbf{T}_{x}^{\left(p_{i}\right)} \overline{\mathbf{F}}^{\left(p_{i}\right)} \mathbf{h}^{(x)}+\mathbf{T}_{y}^{\left(p_{i}\right)} \overline{\mathbf{F}}^{\left(p_{i}\right)} \mathbf{h}^{(y)}+\mathbf{w}^{\left(p_{i}\right)},
$$

where $\mathbf{w}^{\left(p_{i}\right)}=\left[\mathbf{w}_{p_{i}}^{T}, \mathbf{w}_{p_{i}+N}^{T}, \cdots, \mathbf{w}_{p_{i}+\left(L_{h}-1\right) N}^{T}\right]^{T}$. For the two sets of pilots, one can deduce from (10) that

$$
\underbrace{\left[\begin{array}{c}
\mathbf{r}^{\left(p_{1}\right)} \\
\mathbf{r}^{\left(p_{2}\right)}
\end{array}\right]}_{\mathbf{r}}=\underbrace{\left[\begin{array}{cc}
\mathbf{T}_{x}^{\left(p_{1}\right)} \overline{\mathbf{F}}^{\left(p_{1}\right)} & \mathbf{T}_{y}^{\left(p_{1}\right)} \overline{\mathbf{F}}^{\left(p_{1}\right)} \\
\mathbf{T}_{x}^{\left(p_{2}\right)} \overline{\mathbf{F}}^{\left(p_{2}\right)} & \mathbf{T}_{y}^{\left(p_{2}\right)} \overline{\mathbf{F}}^{\left(p_{2}\right)}
\end{array}\right]}_{\mathbf{D}} \underbrace{\left[\begin{array}{l}
\mathbf{h}^{(x)} \\
\mathbf{h}^{(y)}
\end{array}\right]}_{\mathbf{h}}+\underbrace{\left[\begin{array}{l}
\mathbf{w}^{\left(p_{1}\right)} \\
\mathbf{w}^{\left(p_{2}\right)}
\end{array}\right]}_{\mathbf{w}} .
$$

The least square method can then be applied to find the estimated CIR vector $\hat{\mathbf{h}}$ as follows

$$
\hat{\mathbf{h}}=\left(\mathbf{D}^{\dagger} \mathbf{D}\right)^{-1} \mathbf{D}^{\dagger} \mathbf{r}
$$

where $\mathbf{D}$ is a square matrix of size $4 L_{h}$.

\section{B. The proposed preamble design}

An ideal solution to avoid the imperfection of matrix inversion in (12) is to design $\mathbf{D}$ such that

$$
\mathbf{D}^{\dagger} \mathbf{D}=\alpha \mathbf{I}_{4 L_{h}},
$$

where $\alpha$ is a positive real constant. This condition can be easily satisfied thanks to the preamble design. Indeed, let us rewrite $\mathbf{D}=\overline{\mathbf{F}} \mathbf{T}$, where

$$
\mathbf{T}=\left[\begin{array}{ll}
\mathbf{T}_{x}^{\left(p_{1}\right)} & \mathbf{T}_{y}^{\left(p_{1}\right)} \\
\mathbf{T}_{x}^{\left(p_{2}\right)} & \mathbf{T}_{y}^{\left(p_{2}\right)}
\end{array}\right] \quad \text { and } \quad \overline{\mathbf{F}}=\left[\begin{array}{cc}
\overline{\mathbf{F}}^{\left(p_{1}\right)} & \mathbf{0} \\
\mathbf{0} & \overline{\mathbf{F}}^{\left(p_{2}\right)}
\end{array}\right]
$$

In order to meet (13), one can select the preamble pilots so as $t_{x, p_{i}}=\cdots=t_{x, p_{i}+\left(L_{h}-1\right) N}=t_{y, p_{1}}=\cdots=$ $t_{y, p_{1}+\left(L_{h}-1\right) N}=-t_{y, p_{2}}=\cdots=-t_{y, p_{2}+\left(L_{h}-1\right) N}=t_{0}$. Thus,

$$
\mathbf{T}_{x}^{\left(p_{1}\right)}=\mathbf{T}_{y}^{\left(p_{1}\right)}=\mathbf{T}_{x}^{\left(p_{2}\right)}=-\mathbf{T}_{y}^{\left(p_{2}\right)}=t_{0} \mathbf{I}_{2 L_{h}}
$$

It is easy to verify that $\overline{\mathbf{F}} \overline{\mathbf{F}}^{\dagger}=\overline{\mathbf{F}}^{\dagger} \overline{\mathbf{F}}=L_{h} \mathbf{I}_{4 L_{h}}$. Hence, we deduce

$$
\begin{aligned}
\mathbf{D}^{\dagger} \mathbf{D} & =L_{h} \mathbf{T}^{\dagger} \mathbf{T}, \\
& =\underbrace{2 L_{h} t_{0}^{*} t_{0}}_{\alpha} \mathbf{I}_{4 L_{h}} .
\end{aligned}
$$

In that case, the estimated CIR vector reads

$$
\begin{aligned}
\hat{\mathbf{h}} & =\mathbf{h}+\frac{1}{\alpha} \mathbf{D}^{\dagger} \mathbf{w} \\
& =\mathbf{h}+\frac{1}{2 L_{h} t_{0}}\left[\begin{array}{cc}
\mathbf{I}_{2 L_{h}} & \mathbf{I}_{2 L_{h}} \\
\mathbf{I}_{2 L_{h}} & -\mathbf{I}_{2 L_{h}}
\end{array}\right] \overline{\mathbf{F}}^{\dagger} \mathbf{w} .
\end{aligned}
$$

From (16), calculation yields

$$
E\left(\|\mathbf{h}-\hat{\mathbf{h}}\|^{2}\right)=2 \sigma^{2} /\left|t_{0}\right|^{2},
$$

where $\sigma^{2}$ is noise variance. Obviously, the MSE of SP CE can be minimized by maximizing $\left|t_{0}\right|$. In this paper, we propose to design the sparse preamble pilots so as to maximize $\left|t_{0}\right|$. Different from the IAM method, in which the pilots are carefully 
designed for each of sub-carriers, the proposed method aims to design the pilots at the $2 L_{h}$ sub-carriers sparsely distributed throughout the spectrum.

Recall from Section III-A that SP CE uses two sets of pilots (denoted by $\mathcal{S}_{1}, \mathcal{S}_{2}$ ) allocated at the sub-carriers $\left\{p_{i}, p_{i}+N, p_{i}+2 N, \cdots, p_{i}+\left(L_{h}-1\right) N\right\}, i \in\{1,2\}$. Assume 3 CO-OFDM/OQAM symbols (3 time-slots) for preamble in each polarization and the pseudo pilots are counted at the middle time-slot (i.e., $n_{0}=2$ ). On $x$ polarization, we propose to place at the frequency-time positions $\left(p_{i}-1, n_{0}-\right.$ $1),\left(p_{i}-1, n_{0}\right),\left(p_{i}-1, n_{0}+1\right),\left(p_{i}, n_{0}-1\right),\left(p_{i}, n_{0}\right),\left(p_{i}, n_{0}+\right.$ $1),\left(p_{i}+1, n_{0}-1\right),\left(p_{i}+1, n_{0}\right),\left(p_{i}+1, n_{0}+1\right)$ the following preamble pattern

$$
\left[\begin{array}{ccc}
(-j)^{\xi+1} & j^{\xi+1} & (-j)^{\xi+1} \\
-(-j)^{\xi+1} & j^{\xi} & (-j)^{\xi+1} \\
(-j)^{\xi+1} & -j^{\xi+1} & (-j)^{\xi+1}
\end{array}\right]
$$

where $\xi=\bmod \left(p_{i}, 2\right)$ (i.e., $\xi=0$ for even $p_{i}$ and $\xi=1$ for odd $p_{i}$ ), the vertical and horizontal axes stand for the frequency and time dimensions, respectively. This pattern can then be slided along frequency dimension to apply on the sub-carriers $\left(p_{i}+N, p_{i}+2 N, \cdots, p_{i}+\left(L_{h}-1\right) N\right)$ in the set $\mathcal{S}_{i}$. The proposed solution offers $\left|t_{0}\right|=1+2 \beta+2 \gamma+4 \delta$, which is greater than $\left|t_{m}\right|$ achieved by the E-IAM-C method. Note that changing signs of all pilots in (18) results in the same $\left|t_{0}\right|$. Hence, on $y$ polarization, we propose to apply (18) on $\mathcal{S}_{1}$ and the reverse signs of (18) on $\mathcal{S}_{2}$, in order to satisfy the condition in (14). An intuitive illustration of the proposed pattern is shown in Fig. 3.

We conclude this section with two remarks. Firstly, to relax the complexity of SP CE method, the matrix $\mathbf{D}$ can be calculated off-line once and applied for the remaining frames. The actual complexity of SP CE method counts at the multiplication of $\mathbf{D}^{\dagger}$ with $\mathbf{r}$ as shown in (12), which takes $\mathcal{O}\left(16 L_{h}^{2}\right)$ arithmetic operations. The IAM methods require the inversion and multiplication for $M$ matrices of size $2 \times 2$, which take $\mathcal{O}(16 M)$. Since $M=N L_{h}$, the IAM methods thus need $\mathcal{O}\left(16 N L_{h}\right)$ arithmetic operations. Hence, the SP CE is $L_{h} / N$ times more complex than IAM methods depending on the channel length $L_{h}$ and the sparsity $N$ of the pilots. Secondly, we would like to point out that the conventional SP CE was first invented for OFDM and later applied for OFDM/OQAM in radio communications. This direct application did not take into account the IMI and used the conventional SP CE preamble pattern, i.e. $a_{p_{i}, n_{0}}=\cdots=a_{p_{i}+\left(L_{h}-1\right) N, n_{0}}=1$ and zeros elsewhere. In contrast with this conventional SP $\mathrm{CE}$ pilots design, we propose in this paper a new preamble pattern with $\left|t_{0}\right|$ maximization criterion. Therefore, the new preamble design for CO-OFDM/OQAM in this paper could also be straightforwardly applied for OFDM/OQAM in radio communications.

\section{Simulation Results AND Discussions}

This section presents simulation results to validate the proposed preamble for SP CE method. Monte-Carlo simulations have been carried out thanks to the open source MATLAB toolbox Optilux. PHYDYAS prototype filter with overlapping factor 4 is applied. Each CO-OFDM/OQAM frame contains

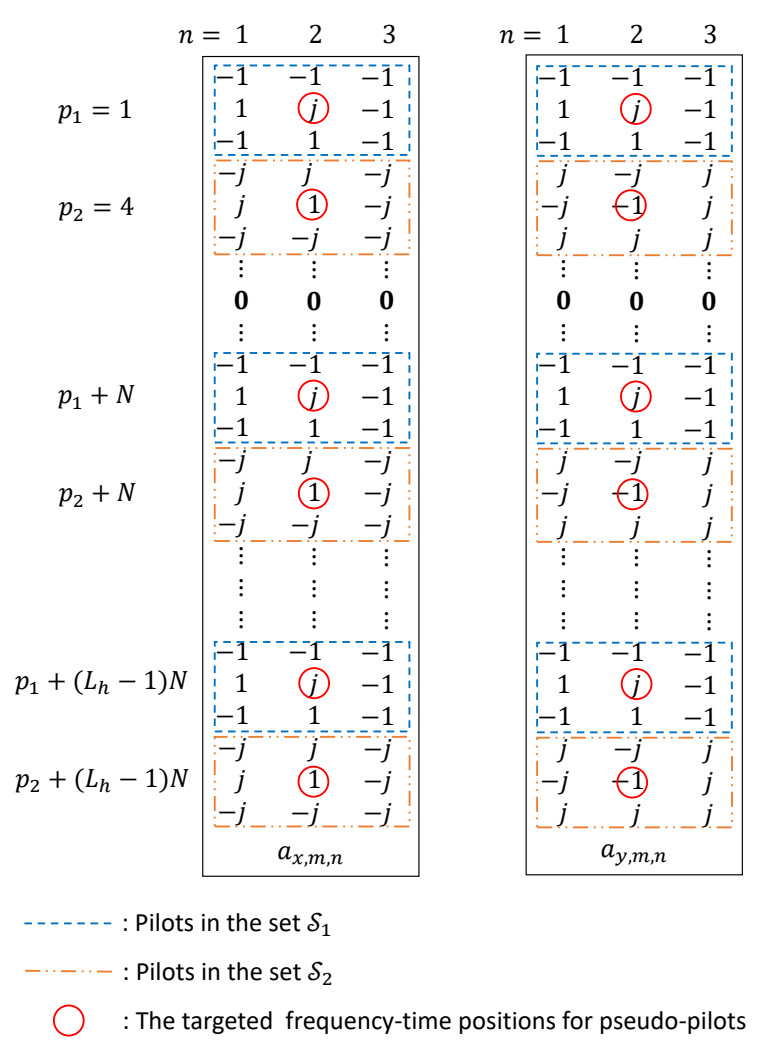

Fig. 3. An example of the proposed preamble pattern for SP CE with $p_{1}=1, p_{2}=4, N$ even, and $n_{0}=2$.

100 symbols including the preambles. A standard single mode fiber (SSMF) transmission line of length $1000 \mathrm{~km}$ is considered here to emulate chromatic dispersion (CD) and polarization mode dispersion (PMD). We do not emulate here fiber loss nor non-linear effects. The fiber dispersion is $17 \mathrm{ps} / \mathrm{km} / \mathrm{nm}$. The first order PMD coefficient is $0.2 \mathrm{ps} / \sqrt{\mathrm{km}}$ [17]. Forward error correction (FEC) code is not considered in this simulation. The optical signal-to-noise ratio (OSNR) is adjusted by feeding appropriate ASE noise at the receiver side through a $3-\mathrm{dB}$ coupler. The OSNR is defined by [18, Eq. (33)]

$$
\text { OSNR }=\frac{P}{2 N_{A S E} B_{\text {ref }}},
$$

where $P$ is the total average signal power summed over the two states of polarization, $N_{A S E}$ is the spectral density of ASE noise in one polarization, and the reference bandwidth $B_{\text {ref }}$ is commonly taken to be $12.5 \mathrm{GHz}$, corresponding to a $0.1 \mathrm{~nm}$ resolution bandwidth of optical spectrum analyzers at $1550 \mathrm{~nm}$ carrier wavelength.

We firstly consider the PDM CO-OFDM/OQAM system with 4-QAM inputs, sampling rate $R_{s}=10$ Gbaud which yields the payload data rate of $37.4 \mathrm{~Gb} / \mathrm{s}, M=512$ subcarriers and $L_{h}=64$. Fig. 4 shows BER performances of the considered system associated with different channel estimation methods. The corresponding average PAPR values of PDM CO-OFDM/OQAM transmitted signal are presented in TABLE I. These PAPR values are measured by taking the ratio between the peak power of preamble divided by the average power of payload. The lower PAPR, the more robust 


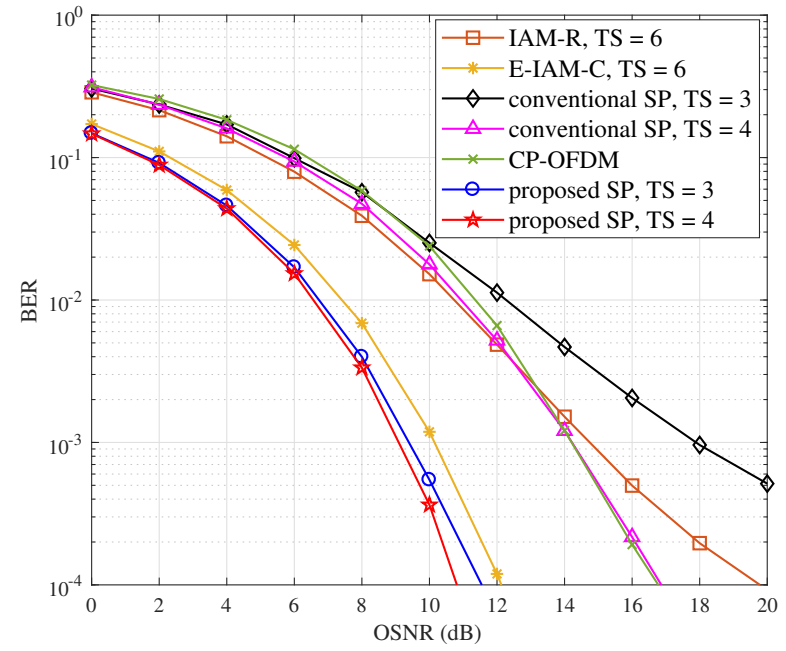

Fig. 4. BER performance of PDM CO-OFDM/OQAM system associated with different channel estimation methods, $R_{s}=10$ Gbaud, $M=512$, 4-QAM.

TABLE I

THE PAPR (IN DB) OF PDM CO-OFDM/OQAM TRANSMITTED SIGNAL ASSOCIATED WITH DIFFERENT CE METHODS, $\mathrm{M}=512$.

\begin{tabular}{|c|c|c|}
\hline CE methods & Time slots (TS) & PAPR \\
\hline IAM-R & 6 & 19 \\
\hline E-IAMC & 6 & 27.3 \\
\hline CP-OFDM with LS & 4 & 8.3 \\
\hline Conventional SP & 3 or 4 & 7 \\
\hline The proposed SP & 3 or 4 & 18.7 \\
\hline
\end{tabular}

PDM CO-OFDM/OQAM systems against nonlinear transfer functions of opto-electronic devices (RF drivers, modulators...) embedded into the transmitter and receiver. Note that a lower PAPR could also be valuable if we took into account fiber non-linear effects, even if it can be relevant to notice that reference [19] shows that sensitivity to fiber non-linearities of OFDM is not directly correlated to PAPR. Furthermore, IAM$\mathrm{R}$ and E-IAM-C always need 6 time-slots (TS) for preamble on each polarization. The proposed sparse preamble (SP) needs 3 TS on each polarization. Thanks to the advantage of SP in terms of TS, one can add one more all-zeros symbol to separate the preamble from data, which results in the SP with 4 TS. Similar technique can be applied for the conventional SP to improve error-rate performance. Note that by adding all-zeros symbol, there is no significant difference in terms of PAPR between SP with 3 and 4 TS.

From Fig. 4 and TABLE I, we observe that the conventional SP with 4 TS not only achieves a gain of roughly $3 \mathrm{~dB}$ at BER $=10^{-4}$, but also offers a lower PAPR compared to the IAM$\mathrm{R}$, which was introduced in [14]. In addition, a significant BER performance improvement can be attained by E-IAM-C, e.g., $5 \mathrm{~dB}$ gain at $\mathrm{BER}=10^{-4}$ compared to the conventional SP with 4 TS. However, this significant gain in terms of BER comes at the price of higher PAPR value. The proposed solution tackles this challenge. Indeed, the proposed SP with 3 and 4 TS respectively achieve the gains of $0.5 \mathrm{~dB}$ and 1 $\mathrm{dB}$ at $\mathrm{BER}=10^{-4}$ compared to E-IAM-C. The PAPR of the proposed SP is significantly lower than the one of E-IAM-

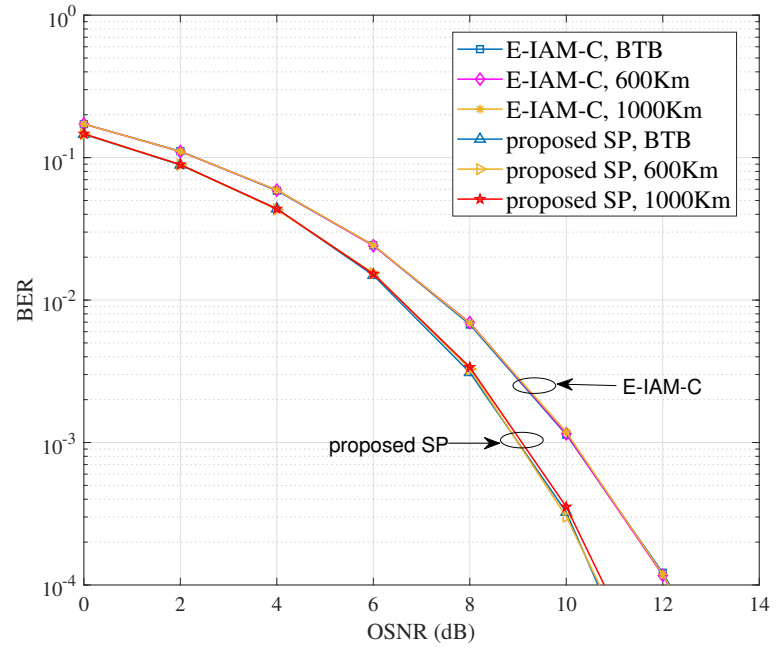

(a) $M=512, L_{h}=64$.

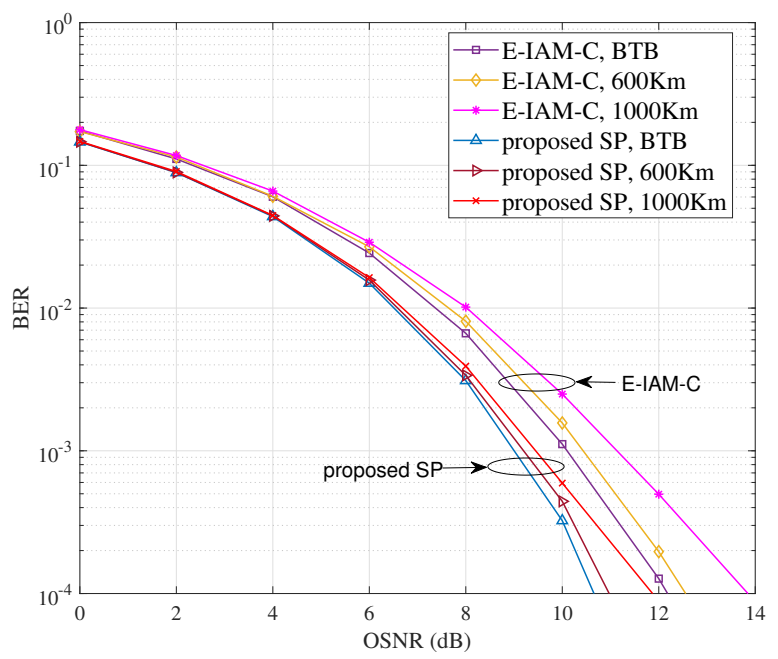

(b) $M=64, L_{h}=32$

Fig. 5. BER performance of PDM CO-OFDM/OQAM system associated with different channel estimation methods vs different distances.

$\mathrm{C}$ and roughly equivalent to the IAM-R one. Note that there is no significant improvement in BER by increasing TS of the SP (i.e., adding more all-zeros symbols between preamble and data). In Fig. 4, the conventional OFDM with cyclic prefix $(\mathrm{CP})$ is also considered as a reference. The $\mathrm{CP}$ ratio is $1 / 8$. The least square (LS) CE is used for CP-OFDM with 2 QAM symbols preamble (i.e., TS $=4$ OQAM time-slots). We observe that the BER performance of CP-OFDM used with LS CE is close to the one of OFDM/OQAM with conventional SP CE. Since this paper focuses on OFDM/OQAM, we prefer to use 2 QAM symbols (TS $=4$ ) as preamble for the LS CE of CPOFDM for a fair comparison in terms of time-slots. However, the performance of LS CE for CP-OFDM can be improved by using time-domain averaging technique with more QAM symbols at the preamble.

In Fig. 5, the E-IAM-C and the proposed SP CE methods, which achieve the better BER performances compared to the others in Fig. 4, are selected to illustrate the performances versus different distances (back-to-back (BTB), 600 km, 1000 


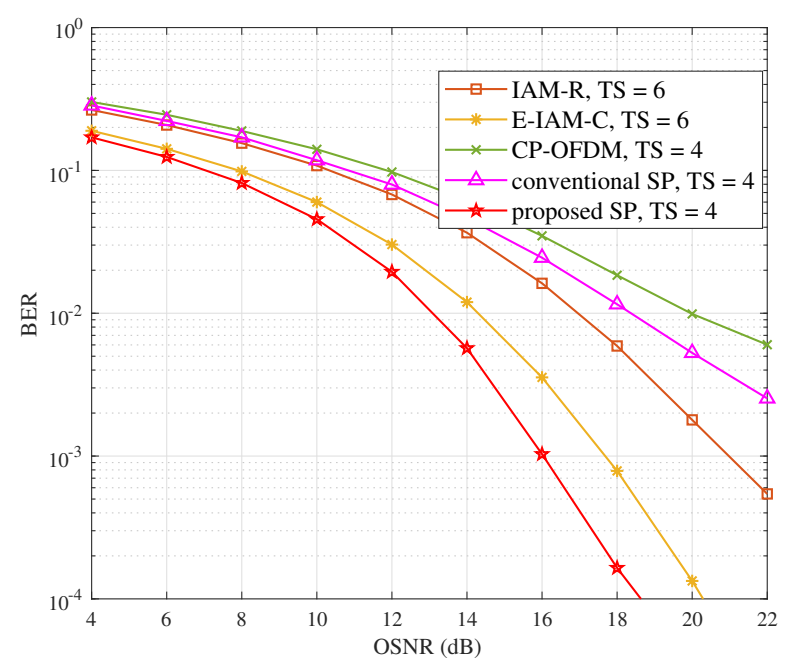

Fig. 6. BER performance of PDM CO-OFDM/OQAM system associated with different channel estimation methods, $R_{s}=10$ Gbaud, $M=512,16-\mathrm{QAM}$.

$\mathrm{km})$. Similar simulation parameters as used for Fig. 4 are considered. With $M=512$ and $L_{h}=64$, we observe from Fig. 5(a) that there is no significant difference in BER of these methods for the considered distances. Since the variation in distance affects the channel delay spread, the explanation for this observation is twofold. Firstly, for the E-IAM-C, which aims to estimate the channel frequency response (CFR), the number of sub-carriers $M=512$ is sufficiently large to ensure flat-fading channels at all sub-carriers for any of the considered distances. Secondly, for the proposed SP method, which aims to estimate the channel impulse response (CIR), the estimated CIR length $L_{h}=64$ is sufficiently large to ensure that there is no underestimation of the CIR length for any of the considered distances. Indeed, to illustrate the BER variation with respect to distance, one can select $M=64$ and $L_{h}=32$ as shown in Fig 5(b). The results in Fig. 5 show that the parameters $M=512$ and $L_{h}=64$ are sufficiently high to ensure the optimal performance at $1000 \mathrm{~km}$ for the considered simulation setup.

Fig. 6 illustrates the BER performance of the COOFDM/OQAM system for 16-QAM inputs with similar setup, i.e. $R_{s}=10$ Gbaud which yields the payload data rate of 74.8 $\mathrm{Gb} / \mathrm{s}$. We observe that the proposed SP method still offers a significant gain compared to the IAM-R method introduced in [14]. In addition, the proposed SP methods achieves a gain of more than $6 \mathrm{~dB}$ compared to the conventional SP at the BER close to $10^{-3}$. The OFDM/OQAM system with conventional SP CE method has a better performance compared to CPOFDM used with least square channel estimation. Let us step forward to consider the PDM CO-OFDM/OQAM system with 4-QAM inputs, sampling rate $R_{s}=32$ Gbaud which yields the payload data rate of $119.6 \mathrm{~Gb} / \mathrm{s}, M=2048$ sub-carriers and $L_{h}=256$. We observe from Fig. 7 that the proposed SP with 4 TS still offers a gain in BER compared to E-IAM-C. However, the gain is less significant in this case $(0.5 \mathrm{~dB}$ gain at $\mathrm{BER}=10^{-4}$ compared to $1 \mathrm{~dB}$ gain at the same BER as shown in Fig. 4). This is due to the increasing of sampling rate, which leads to the increasing of $L_{h}$. The proposed SP achieves

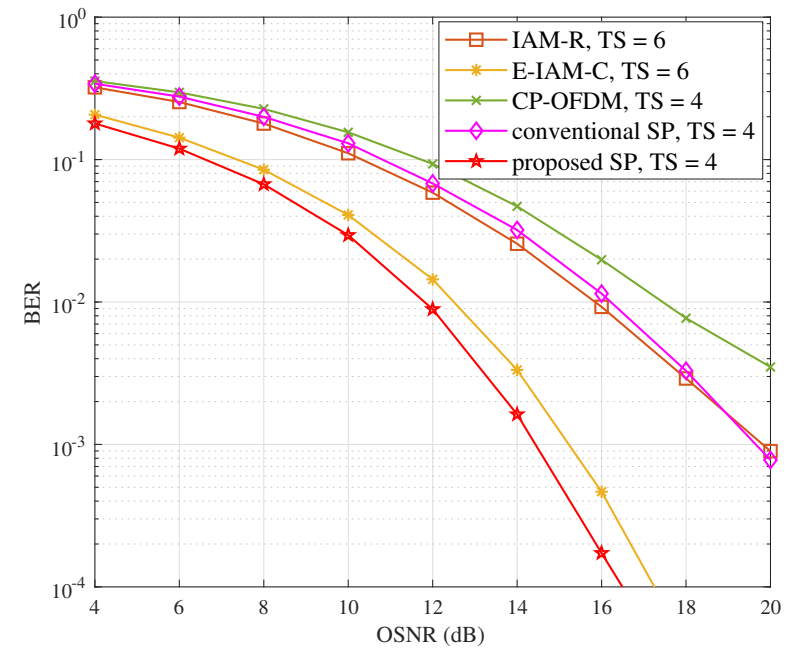

Fig. 7. BER performance of PDM CO-OFDM/OQAM system associated with different channel estimation methods, $R_{s}=32$ Gbaud, $M=2048$, 4-QAM.

significant gains of roughly $6 \mathrm{~dB}$ and $7 \mathrm{~dB}$ at $\mathrm{BER}=10^{-4}$ compared to the conventional SP and the IAM-R, respectively. The average PAPR of the transmitted signal is increased by increasing $M$. However, the difference in terms of PAPR of the considered methods remains the same. Indeed, as shown in TABLE I, the conventional SP still offers the lowest PAPR, while the E-IAM-C results in the highest PAPR. The proposed SP secures a PAPR that is lower than the one reached by EIAM-C and equivalent to the one offered by IAM-R.

The estimated CIR $L_{h}$ is proportional to the link length and the sampling frequency. In simulation, $L_{h}$ can be easily predicted by plotting the CIR of the simulated channel. However, the link length might be unknown in practice due to the reconfiguration of wavelength paths. Therefore, at a given sampling frequency, we propose to firstly simulate and store in a look-up table (LUT) the $L_{h}$ values corresponding to the reference BER and OSNR. Consequently, for practical application with unknown fiber length, one can establish the communications by tuning $L_{h}$ based on the LUT until it reaches the targeted BER. The tuned value of $L_{h}$ can be used for the whole communication session. By storing the fiber length associated with each $L_{h}$ and the reference BER, one could also estimate partly the fiber length by using this method.

\section{CONClusion}

The sparse preamble channel estimation method was exploited for PDM CO-OFDM/OQAM systems. A novel design for the sparse preamble was proposed. In comparison with IAM methods, the proposed solution has several advantages. Firstly, the proposed SP achieves a significant gains in terms of BER performance compared to both IAM-R and E-IAM$\mathrm{C}$ methods. Secondly, the proposed SP gives a lower PAPR compared to E-IAM-C and an equivalent PAPR compared to IAM-R. Last but not least, the proposed SP offers a low preamble overhead by using only 3 or 4 time-slots compared to 6 time-slots as required by IAM methods. With robustness against ASE noise, the proposed SP is a promising solution for 
PDM CO-OFDM/OQAM channel estimation. In addition, the proposed SP was generally presented in baseband model before applying to PDM CO-OFDM/OQAM systems. Therefore, it can be straightforwardly applied to radio communications. This paper focused on the algorithm and preamble design for the SP CE. The phase noise and fiber non-linear effects will be investigated in future works including experimental implementation.

\section{ACKNOWLEDGEMENT}

The authors would like to thank the Editor and the anonymous reviewers for their constructive feedback. The first author would also like to acknowledge the helpful discussions with Dr. Trung-Hien Nguyen at Université Libre de Bruxelles. This work was partly funded by the Brittany Region under the grant number SAD 2015 - ULTRACOM (9293).

\section{REFERENCES}

[1] Z. Zheng, F. Frey, P. W. Berenguer, and J. K. Fischer, "Low-complexity equalization scheme for multicarrier offset-QAM systems," IEEE Photon. Technol. Lett., 2017.

[2] T.-H. Nguyen and C. Peucheret, "Kalman filtering for carrier phase recovery in optical offset-QAM Nyquist WDM systems," IEEE Photon. Technol. Lett., vol. 29, no. 12, pp. 1019-1022, 2017.

[3] X. Fang and F. Zhang, "Phase noise estimation and suppression for PDM CO-OFDM/OQAM systems," IEEE J. Lightw. Technol., vol. 35, no. 10, pp. 1837-1846, 2017.

[4] J. Zhao, "DFT-based offset-QAM OFDM for optical communications," Opt. Express, vol. 22, no. 1, pp. 1114-1126, 2014.

[5] J. Fickers, A. Ghazisaeidi, M. Salsi, G. Charlet, P. Emplit, and F. Horlin, "Multicarrier offset-QAM for long-haul coherent optical communications," IEEE J. Lightw. Technol., vol. 32, no. 24, pp. 4069-4076, 2014.

[6] P. Siohan, C. Siclet, and N. Lacaille, "Analysis and design of OFDM/OQAM systems based on filterbank theory," IEEE Trans. Signal Process., vol. 50, no. 5, pp. 1170-1183, 2002.

[7] T.-H. Nguyen, F. Rottenberg, S.-P. Gorza, J. Louveaux, and F. Horlin, "Efficient chromatic dispersion compensation and carrier phase tracking for optical fiber FBMC/OQAM systems," IEEE J. Lightw. Technol., vol. 35, no. 14, pp. 2909-2916, 2017.

[8] F. Rottenberg, T.-H. Nguyen, S.-P. Gorza, F. Horlin, and J. Louveaux, "Advanced chromatic dispersion compensation in optical fiber FBMCOQAM systems," IEEE Photonics Journal, vol. 9, no. 6, pp. 1-10, 2017.

[9] Y. Yu, P. D. Townsend, and J. Zhao, "Equalization of dispersion-induced crosstalk in optical offset-QAM OFDM systems," IEEE Photon. Technol. Lett., vol. 28, no. 7, pp. 782-785, 2016.

[10] J. Zhao, "Channel estimation in DFT-based offset-QAM OFDM systems," OSA Optics Express, vol. 22, no. 21, pp. 25 651-25 662, 2014.

[11] D. Wang, L. Yuan, J. Lei, S. Li, R. Ding, D. Wang et al., "Joint channel/frequency offset estimation and correction for coherent optical FBMC/OQAM system," Elsevier Optical Fiber Technology, vol. 39, pp. 87-94, 2017.

[12] C. Lélé, J.-P. Javaudin, R. Legouable, A. Skrzypczak, and P. Siohan, "Channel estimation methods for preamble-based OFDM/OQAM modulations," Eur. Trans. Telecomms., vol. 19, no. 7, pp. 741-750, 2008.

[13] E. Kofidis and D. Katselis, "Improved interference approximation method for preamble-based channel estimation in FBMC/OQAM," in EUSIPCO, 2011, pp. 1603-1607.

[14] X. Fang, Y. Xu, Z. Chen, and F. Zhang, "Frequency-domain channel estimation for polarization-division-multiplexed CO-OFDM/OQAM systems," IEEE J. Lightw. Technol., vol. 33, no. 13, pp. 2743-2750, 2015.

[15] - "Time-domain least square channel estimation for polarizationdivision-multiplexed CO-OFDM/OQAM systems," IEEE J. Lightw. Technol., vol. 34, no. 3, pp. 891-900, 2016.

[16] B. Lin, X. Tang, Y. Li, S. Zhang, and Z. Ghassemlooy, "Dualpolarization OFDM/OQAM-PON with efficient channel equalization methods," in IEEE Opto-Electronics and Communications Conference (OECC) and Photonics Global Conference (PGC), 2017, 2017, pp. 1-4.

[17] F. Horlin, J. Fickers, P. Emplit, A. Bourdoux, and J. Louveaux, "Dualpolarization OFDM-OQAM for communications over optical fibers with coherent detection," Opt. Express, vol. 21, no. 5, pp. 6409-6421, 2013.
[18] R.-J. Essiambre, G. Kramer, P. J. Winzer, G. J. Foschini, and B. Goebel, "Capacity limits of optical fiber networks," IEEE J. Lightw. Technol., vol. 28 , no. 4 , pp. 662-701, 2010.

[19] M. Song, E. Pincemin, B. Baeuerle, A. Josten, D. Hillerkuss, J. Leuthold, and I. Tomkos, "Fibre nonlinearity limitations of 1 Tbps (10x100 Gbps) multi-band e-OFDM super-channel," in OSA Signal Processing in Photonic Commun., 2016, pp. SpW1G-2.

Nhat-Quang Nhan (M'17) received the B.Eng. and M.Eng. degrees in electrical engineering from International University-Vietnam National University of Hochiminh City, Vietnam, in 2010 and 2012, respectively, the M.Sc. degree in electronics and telecommunications engineering from the University of Rennes 1, France, in 2013, and the Ph.D. degree in digital communications engineering from the University of Brest, France, in 2016. He was a Visiting Scholar with the Memorial University of Newfoundland, Canada, in 2014, and Missouri S\&T University, USA, in 2015. He is currently a researcher with the Lab-STICC CNRS UMR 6285, France. His research interests focus on signal processing and coding theory for wireless and optical communications.

Pascal Morel was born in Rennes, France, in 1980. He received the M.Eng. and M.S. degrees from the École Nationale d'Ingénieurs de Brest in 2003 and Ph.D. degree from the Université de Bretagne Occidentale, Brest in 2006. In 2008, he joined the École Nationale d'Ingénieurs de Brest, France (ENIB) as Associate Professor (Maître de Conférences). Since then, his main field of interest have been in the area of high speed optical transmission systems, mainly focused on semiconductor optical amplifiers modeling and applications.

Stéphane Azou (SM'17) was born in 1970. He received the M. Sc. degree in electronics, and the Ph.D. degree both from the University of Brest (UBO), Brest, France, in 1993, and 1997, respectively. He also received the HDR degree (accreditation to supervise research) from the same university in may 2007. From 1998 to 2000, he was with the Signal Processing Group at the French Naval Academy, Lanvéoc, France. From 2000 to 2012, he has been an Associate Professor at the UBO where he developed research activities related to spread-spectrum techniques, communications intelligence and nonlinear filtering. From 2007 to 2012, he headed the Signal processing for communications research team in the Lab-STICC laboratory (CNRS, UMR 6285). In September 2012, he joined the École Nationale d'Ingénieurs de Brest, France (ENIB) as a Full Professor and he is currently the head of Devices and Multiphysics Interfaces (DIM) team within the Lab-STICC. His current research interests include fiber optics communication, behavioral modeling of optoelectronic devices, signal processing for communications and linearization techniques. He has coauthored more than 60 journal and conference scientific papers.

Michel Morvan was born in Quimper, France in 1965. He received the diplôme d'ingénieur degree from the Ecole Nationale Supérieure des Télécommunications de Bretagne in 1988. He joined the France Telecom Research Centre (formerly Centre National des Etudes de Telecommunications) in Lannion, France, in 1989 where he worked successively on Optical Coherent Transmission, High capacity WDM systems and SDH/WDM networking. From 2000 to 2002, he then worked at Sycamore Network as a senior network architect. In 2002, he joined the Institut Telecom as a senior lecturer at TELECOM Bretagne, Brest France. He lectures on optical components and transmission systems, system design and simulation as well as optical networking. His current research interests are focused on optical transmission flexibility issues and optical core/metro network architectures.

Philippe Gravey graduated from the Ecole Polytechnique (Palaiseau - France) in 1978, from the Ecole Nationale Supérieure des Télécommunications (Paris - France) in 1980 and received a Diplôme d'Etudes Approfondies in Optics (Orsay - France), also in 1980. He then joined the CNET (now Orange Labs) in Lannion to work on holographic techniques and materials applied to optical interconnections. Between 1993 and 1997, he was in charge of the "Photonic Switching Processors" department. In 2000, he joined the Optics Department of TELECOM Bretagne and co-founded the Optogone company in 2001, which developed liquid crystal based devices or sub-systems for WDM applications. He returned to Telecom Bretagne in 2005. His present research interests optical metro and core networks, data center networks, optical burst/packet switching and transponders for flexgrid networks. He authored or co-authored more than 140 international publications or communications. 
Erwan Pincemin graduated from Ecole Supérieure d'Optique (SupOptique), Orsay, France and from Université Paris XI, Orsay, France in "Optics and Photonics" both in 1996. In 1997, he joined Alcatel Alsthom Recherche, Marcoussis, France where he worked on dispersion-managed soliton propagation and optical regeneration for $40 \mathrm{Gbps}$ WDM submarine transmission systems. In 2000, he is enrolled by France Telecom R\&D, Lannion, France where he worked on high-speed optical terrestrial transmission systems at 10 Gbps, 40Gbps and beyond. He participated to several European collaborative projects (NOBEL I \& II, e-Photon-One,...). More recently, in Orange Labs, Lannion, France, he is in charge of research on $100 \mathrm{Gbps}$ and beyond optical transmissions, including advanced modulations, coherent detection, digital mitigation of fiber impairments, and has also some key contributions on flexible optical networking, in particular through his participation to European FOX-C and CELTIC SASER consortia. He is currently involved in ITU-T standardization process through his activities on interworking of $100 \mathrm{Gbps}$ and beyond optical interfaces in Study Group 15. He is author and coauthor of more than 130 journal and conference papers and 27 patents. Erwan Pincemin is sub-committee chair of the Conference on Lasers and ElectroOptics (CLEO) Europe and participated from 2007 to 2010 and again since 2017 to the technical program committee of the Optical Fiber Communications Conference (OFC). 